\title{
Theoretical Study of Anomalous Forces Externally Induced by Superconductors
}

\author{
Elio B. Porcelli, Victo S. Filho
}

H4D Scientific Research Laboratory, São Paulo, Brazil

Correspondence to: Elio B. Porcelli, Elioporcelli@h4dscientific.com

Keywords: Superconducting Ceramics, Gravitational Shielding, Anomalous Forces, Quantum Entanglement

Received: August 17, $2017 \quad$ Accepted: September 11, $2017 \quad$ Published: September 14, 2017

Copyright (C) 2017 by authors and Scientific Research Publishing Inc.

This work is licensed under the Creative Commons Attribution International License (CC BY 4.0).

http://creativecommons.org/licenses/by/4.0/

\section{c) (i) Open Access}

\section{ABSTRACT}

In this work we present a theoretical explanation for the possible anomalous forces induced by superconducting disks and toroids, based on the hypothesis of a preexisting state of generalized quantum entanglement that can produce momentum variation exchanged between Cooper pairs and outer particles. Considering the immense amount of particles involved in the phenomenon as coherent Cooper pairs, and indications of previous studies, we use classical quantities as macroscopic observables in our calculations. We here analyzed the behavior of such superconductors and compared the experimental results early obtained in the literature with our theoretical proposal. We found that the theoretical calculations agreed with very good accuracy for two different experiments and devices. The present work really highlights the possibility of superconducting materials to be applied to induce outer forces in the environment and in external objects, as explained by our theoretical model.

\section{INTRODUCTION}

In an intriguing work [1], the authors reported experimental evidence of a gravitational impulse in the local vicinity of charged high- $\mathrm{T}_{c}$ ceramic superconductor $\mathrm{YBa}_{2} \mathrm{Cu}_{3} \mathrm{O}_{7-\mathrm{y}}$ which could induce the deflection of a simple pendulum of several materials. In other work [2], it was also reported the existence of weak gravitation shielding properties of the superconductor $\mathrm{YBa}_{2} \mathrm{Cu}_{3} \mathrm{O}_{7-\mathrm{x}}$ below $70 \mathrm{~K}$ under the applications of high electromagnetic fields. The experimental procedure described in [1] was improved and implemented by same authors in an experimental setup established for the investigation of high voltage discharges in low pressure gases through large superconductors, as published in [3]. The phenomenon is characterized by the existence of apparent anomalous forces in the vicinity of high- $\mathrm{T}_{\mathrm{c}}$ superconductors under nonequilibrium conditions. The results of the improved apparatus confirmed the existence of the unexpected physical interaction proportional to the mass of the objects and independent on their composition in temperature down to $40 \mathrm{~K}$. In other words, within the errors of the measurements it behaves like a gravitational interaction, an effect that cannot be explained in the frame of the known theories as the 
general relativity. The novel effect found from that impulse gravity generator motivated the interest of other researchers that implemented the replication of the experiments in their works, as in $[4,5]$.

A perspective of theoretical explanation for such a phenomenon of gravitational modifications has been proposed in Solomon's works [6-8], in which the experiments in [1] and [2] and their effects were systematically reviewed. As contraposition to those works, other groups also studied the phenomenon and or tried to reproduce the same results, but they were controversial and not conclusive [7-9]. Some theoretical works have investigated more profoundly the possibility of gravitational screening or gravitationalshielding by superconductors $[10,11]$. So, the phenomenon still remains up to date open and needs a more accurate verification.

The connection between gravitational field and superconductivity has been object of study of a lot of works for many years [12-27] due to be a very interesting and novel field of research. However, in the specific line of research concerning to the strange effect here described, we can mainly emphasize works that consider possible explanations by using the general relativity [28-30]. In a 1996 work [28], the author tried to explain the phenomenon by means of a formalism of a quantum theory of general relativity, but it was not possible to obtain results for comparison with the experimental data due to its complexity. Other possibilities tried were to explain the effect by means of generalized Maxwell equations that simultaneously treated weak gravitational and electromagnetic fields $[29,30]$ by considering the weak field approximation for Einstein equations [31, 32]. In a sequence of works in the field, G. Modanese also presented studies concerning to possible alternative explanations to the effect [33-37].

The phenomenon of connection between gravity and superconductivity has been investigated even in very recent works $[38,39]$, indicating the present relevance of the subject.

In this work, we propose a different theoretical explanation of the effect in comparison with other theories. We consider that the state of generalized quantum entanglement (GQE) is preexisting and it rules all particles in the universe. Its effect is usually extremely weak because of the huge entropy involving the mixture of the states of the myriad of particles existing in the universe. For example, if we measure the quantum state of two random particles we cannot detect any correlation between them even considering GQE formalism. In order to rise the effects of GQE state we need to put a huge quantity of particles in the same quantum state locally together such as made in case of internal Cooper pairs of the superconductor material below the critical temperature.

Any N-body calculation is very complex using the quantum mechanics framework regarding the huge quantity $\mathrm{N}$ of microscopic quantum systems such as Cooper pairs. In general lines, our approach consists in considering valid the connection between quantum microscopic quantities and macroscopic observables, based on the theoretical framework of GQE state, as already proposed in recent studies considering other anomalous effects in capacitors [40-42], magnetic cores [43], piezoelectric materials [44] and semiconductors laser diodes [45]. It is relevant to emphasize that such a macro-entanglement state can only take place under very special or circumstances or extreme physical conditions, as in the cases of high voltages or high powers applied to the devices, as presented in the cited experiments. We here found that our theoretical calculations agreed with very good accuracy for two different experiments involving superconductor devices. Such results really reinforce the consistency of GQE hypothesis.

In the next section, we give more details about Podkletnov experiments and describe the model proposed in order to explain the anomalous effects reported in [1-3].

\section{THEORETICAL FRAMEWORK}

\subsection{Experiments in Superconducting Disks}

In [1], it was reported a successful implementation of an impulse gravity generator based on high tension discharges applied on a superconducting ceramic $\mathrm{YBa}_{2} \mathrm{Cu}_{3} \mathrm{O}_{7-\mathrm{y}}$. We here propose a new theoretical model in order to quantify the magnitude of the anomalous forces generated by the operation of that superconductor.

In order to understand the model, we need to describe in more details the features of the anomalous 
effect. The work reports an experiment involving two large two-layer disks, in which one was superconductor (diameter $120 \mathrm{~mm}$ and thickness from $4 \mathrm{~mm}$ to $8 \mathrm{~mm}$ ) and another one an ordinary conductor(diameter $100 \mathrm{~mm}$ and thickness $15 \mathrm{~mm}$ ) in which was applied high-voltage in the range $500 \mathrm{kV}$ to 2 MV. The superconducting material (ceramic) was in the superconducting state, as its temperature was kept below the critical limit $(40 \mathrm{~K})$. During the time interval of high voltage application $(0.1 \mathrm{~ms}$ to 0.01 $\mathrm{ms})$, an impulse was generated in direction to a spherical rubber mass $(18.5 \mathrm{~g})$ connected in the free extremity of a simple pendulum. The pendulum suffered a visible deflection and as the test mass in its extremity was made by rubber the impulse had not electromagnetic character. In the Figure 1, we show a scheme of the apparatus used in the experiment and the effect suffered by the simple pendulum.

The geometric projection of the impulse occurs from the area of the superconducting disk and there was not perceivable decay up to at least a distance of $150 \mathrm{~m}$, even across physical obstacles. As already described, the properties of such an interaction were not explainable by means of any actual theory. Although the effect cannot be completely understood by any actual theory or even by alternative recent theoretical proposals, we assert that it can be surprisingly explained so qualitatively as quantitatively with basis on the new concepts presented from now on. Next we present the numerical approach and calculate the magnitude of such forces by directly adopting the experimental data provided by [1-3].

In a voltage of $2 \mathrm{MV}$, the peak of the current was about $10,000 \mathrm{~A}$, the total charge discharged was 0.1 $C$ (the discharge was provided by a bank of capacitors) and the discharge time remained between $10^{-4} \mathrm{~s}$ and $10^{-5} \mathrm{~s}$. All of the data here described can be found in [1]. The average time in the calculations is $t=5 \times$ $10^{-5} \mathrm{~s}$. As the average electric current is the total charge by the average time, then for that operation time considered the average current in that voltage is $I=0.1 C /\left(5 \times 10^{-5} \mathrm{~s}\right)=2000 \mathrm{~A}$.

A study related to the longitudinal forces proportional to the square of the electric current value was developed according to [46] and these calculations can be summarized by the formula:

$$
F=\frac{\mu_{0} I^{2} L}{4 \pi}
$$

in which $F$ is the longitudinal force (in N), $I$ is the magnitude of the electric current flowing in the circuit, $L$ is the geometric coefficient and $\mu_{0}$ is the magnetic constant $4 \pi \times 10^{-7} \mathrm{H} / \mathrm{m}$.

The charge carriers (electrons) of the electric current pulse move inside the circuit with some drift velocity according to the transitory local potential (electric) and, as result, a nonlocal longitudinal force in

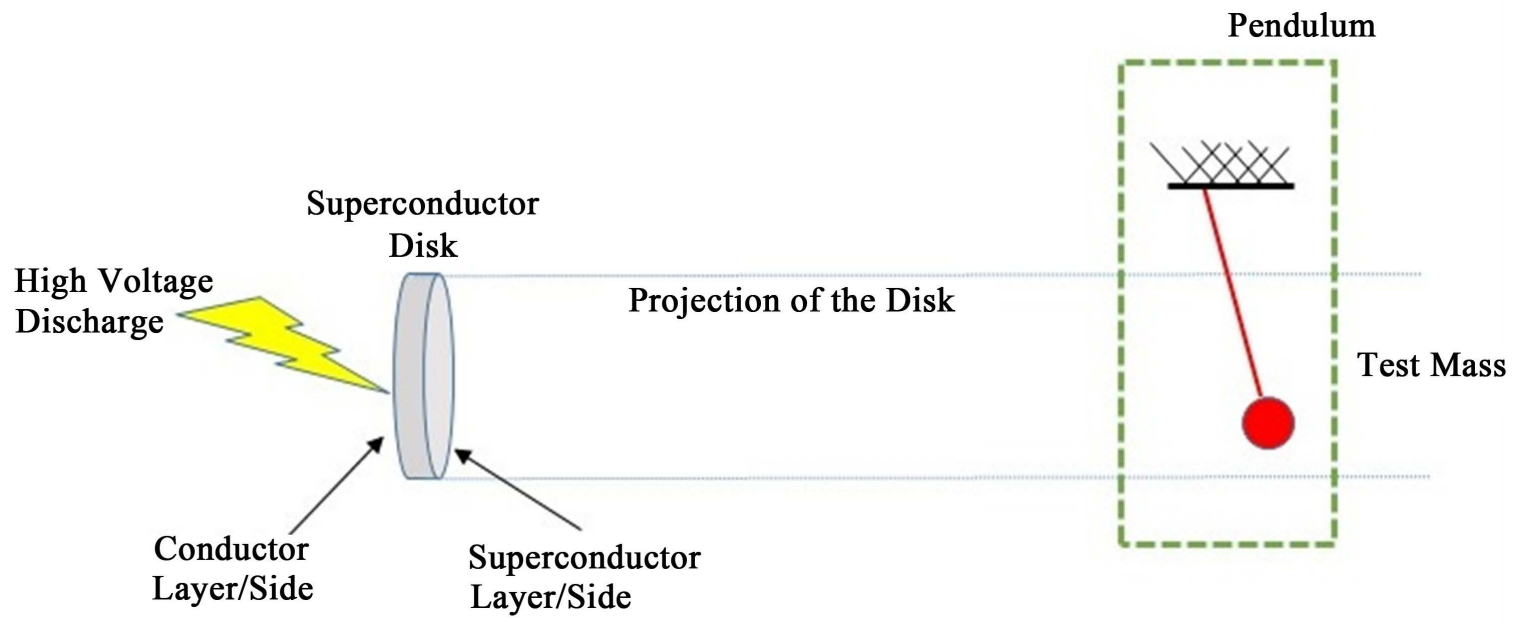

Figure 1. Scheme of the apparatus used in the experiments reported in [1]. The super-conducting ceramic disk subjected to high voltages generates a field of forces which resembles a weak gravitational field in its surroundings that causes the deflection of the mass in the extremity of the simple pendulum. 
the electric current direction is inducted abroad considering their precondition of generalized quantum entanglement with the environment.

The external induction is not intermediated by a magnetic field (photons), then we conjecture that the geometric coefficient (dimensionless) can have its value equal to unity $(L / 4 \pi=1)$ since it depends only on the geometry of the circuit. The nonlocal force equation can be derived from Equation (1) as follows:

$$
F_{m}=\mu_{0} I^{2}
$$

We have to point out that this same equation can be used to calculate the magnitude of the nonlocal force induction generated by the charge carriers inside a resonant cavity of laser diodes, making the gain equal to zero, according to our previous work [41].

The average current is $i=2000 \mathrm{~A}$ for an applied voltage $2 \times 10^{6} \mathrm{~V}$, so that $F_{m}(2 \mathrm{MV})=4 \pi \times 10^{-7} \times(2000)^{2}=5.03 \mathrm{~N}$. For the voltage $10^{6} \mathrm{~V}$, the average current must be $I=1000 \mathrm{~A}$ and, then, we have the force $F_{m}(1 \mathrm{MV})=4 \pi \times 10^{-7} \times(1000)^{2}=1.26 \mathrm{~N}$. For the voltage $5 \times 10^{5} \mathrm{~V}$, the average current must be $I=500 \mathrm{~A}$, so that $F_{m}(500 \mathrm{kV})=4 \pi \times 10^{-7} \times(500)^{2}=0.315 \mathrm{~N}$.

We assert that there are the possibility of particles affects the momenta of others which they are quantically entangled with when subjected to local forces. In other words, beside the interaction among the particles via local forces there is also interaction via nonlocal forces in the case of quantically entangled particles.

It is supposed as basic premise in the generalized quantum entanglement (GQE) framework that all particles are quantically entangled in a generalized way [43]. Therefore the pairs of Cooper in the superconducting state (below the critical temperature) in the interior of a superconducting disk can affect the momentum of the particles that constitute external objects, as the mass of a simple pendulum.

Here such a hypothesis deserves some comments concerning to Cooper pairs. There has been a big controversy in studies related to the mechanism of generation of the superconducting effect in the case of high- $\mathrm{T}_{\mathrm{c}}$ superconductors, that is, if the effect is really caused by coupling of electrons in pairs as occurs in metallic superconductors. In [47], it was reported that many materials are not well described by the BCS model, but the pairing theory is a wider concept in which fermions can be described by means of an effective attractive interaction that produces condensates with large overlaps between pairs. Hence, the BCS concept is not excluded as a possible explanation of the behavior of non-metallic superconductors.

In other work [48], it was studied the mechanism responsible for superconductivity in high-temperature cuprates and reported that evidences indicated that the pairing state could exist but in a new form, characterized by an anisotropic order parameter. Besides it was reported that a class of experiments involving the most widely studied cuprate $\left(\mathrm{YBa}_{2} \mathrm{Cu}_{3} \mathrm{O}_{7-\mathrm{y}}\right)$ and based on the interference of the quantum-mechanical phases in Josephson tunnel junctions and dc SQUID devices gave strong evidence for pairing in a channel with d-wave symmetry. Besides, more recently, although for a different high- $\mathrm{T}_{\mathrm{c}}$ superconductors (BSCCO), it has been also reported in [49] that the coupling electron-electron would be strong enough in order to be responsible by the superconducting effect, although the role of phonons, the glue in the case of conventional Cooper pairs, remains controversial. Further other recent work [50] also suggests the presence of the mechanism of Cooper-pairing in layered high- $\mathrm{T}_{\mathrm{c}}$ superconductors. In other words, by considering all the works that reinforce the presence of electron pairing in high- $\mathrm{T}_{\mathrm{c}}$ superconductors, we really adopt such a hypothesis in our model and indistinctly call them Cooper pairs from now on.

The collective set of Cooper pairs behaves as a single composed particle, due to its dependence under the same wave function despite its macroscopic distribution in the interior of the superconducting disk. The momenta of the particles that compose the pendulum mass are affected when the pairs of Cooper are subjected to a transient longitudinal force in the average time $5 \times 10^{-5} \mathrm{~s}$ when the intense average current is applied. The impulse generated in the pendulum mass is the kinetic reaction of the Cooper pairs subjected to a longitudinal force originated from the high voltage discharge.

The nonlocal force induced by Cooper pairs acts from the area of the superconducting disk $A_{1}$ with diameter $120 \mathrm{~mm}$ and $113.1 \mathrm{~cm}^{2}$ according to $P=F_{m} / A_{1}$, in which $P$ is the induced nonlocal pressure. 
Such a pressure is directly proportional to the longitudinal force $F$ that affects Cooper pairs in a transient way. So, we have the following values for the pressure $P$ to each different applied longitudinal force:

$$
\begin{aligned}
& P(2 \mathrm{MV})=5.03 / 113.1=0.0445 \mathrm{~N} / \mathrm{cm}^{2} ; \\
& P(1 \mathrm{MV})=1.26 / 113.1=0.0111 \mathrm{~N} / \mathrm{cm}^{2} ; \\
& P(500 \mathrm{kV})=0.315 / 113.1=0.0027 \mathrm{~N} / \mathrm{cm}^{2} .
\end{aligned}
$$

The pendulum mass in equilibrium had half spherical area $A_{2}=13.52 \mathrm{~cm}^{2}$ which is achieved by the induction field that emerges from Cooper pairs distributed in the interior of the superconducting disk. The value of the induced force for each value of voltage is calculated in the following way:

$$
\begin{aligned}
& F(2 \mathrm{MV})=P(2 \mathrm{MV}) \times A_{2}=0.0445 \times 13.52=0.6 \mathrm{~N} ; \\
& F(1 \mathrm{MV})=P(1 \mathrm{MV}) \times A_{2}=0.0111 \times 13.52=0.15 \mathrm{~N} ; \\
& F(500 \mathrm{kV})=P(500 \mathrm{kV}) \times A_{2}=0.0027 \times 13.52=0.0365 \mathrm{~N}
\end{aligned}
$$

The longitudinal force generates the dislocation of Cooper pairs and as consequence, since that Cooper pairs and pendulum mass are entangled, they induce a dislocation to the mass of the pendulum, that is, one causes a deflection. In other words, part of the energy (work) that the longitudinal force provided to Cooper pairs moved them in such a way that they induced a dislocation of the pendulum mass, which they were entangled with, by our initial hypothesis. That energy can be calculated by multiplying the induced force by the thickness of the superconducting disk. The thickness of the disk determines the limit or the boundary condition for Cooper pairs-that can be understood as a composed particle distributed in macroscopic dimensions in the interior of the superconducting disk. So, adopting those classical parameters and purely kinetic arguments in the calculation is justified. The work realized by the force for each different voltage and thickness is shown in each of the following calculations:

$$
\begin{aligned}
& E(2 \mathrm{MV}-8 \mathrm{~mm})=0.6 \times 8 \times 10^{-3}=48 \times 10^{-4} \mathrm{~J} ; \\
& E(1 \mathrm{MV}-8 \mathrm{~mm})=0.15 \times 8 \times 10^{-3}=12 \times 10^{-4} \mathrm{~J} ; \\
& E(500 \mathrm{kV}-8 \mathrm{~mm})=0.0365 \times 8 \times 10^{-3}=3 \times 10^{-4} \mathrm{~J} ; \\
& E(2 \mathrm{MV}-4 \mathrm{~mm})=0.6 \times 4 \times 10^{-3}=24 \times 10^{-4} \mathrm{~J} ; \\
& E(1 \mathrm{MV}-4 \mathrm{~mm})=0.15 \times 4 \times 10^{-3}=6 \times 10^{-4} \mathrm{~J} ; \\
& E(500 \mathrm{kV}-4 \mathrm{~mm})=0.0365 \times 4 \times 10^{-3}=1.5 \times 10^{-4} \mathrm{~J}
\end{aligned}
$$

Those values are consistent with the values of the potential energy variation $E_{P}$ of the pendulum as function of the applied voltage and different values of thickness of the dielectric, as indicated in the paper. Such values are shown in Table 1.

Table 1. Values of the potential energy variation $E_{P}$ (in Joules) in function of the voltage applied to the superconductor (in Volts) and its thickness (in $\mathrm{mm}$ ).

\begin{tabular}{ccc}
\hline Voltage $(\mathrm{MV})$ & Thickness $(\mathrm{mm})$ & Potential Energy Variation $E_{P}(\mathrm{~J})$ \\
\hline 2 & 8 & $23.1 \times 10^{-4}$ \\
1 & 8 & $13.9 \times 10^{-4}$ \\
0.5 & 8 & $3.6 \times 10^{-4}$ \\
2 & 4 & $13.1 \times 10^{-4}$ \\
1 & 4 & $8.3 \times 10^{-4}$ \\
0.5 & 4 & $1.8 \times 10^{-4}$ \\
\hline
\end{tabular}


According to the authors in [1], the geometry of the induction region remained unchanged up to distances of the order of $150 \mathrm{~m}$ from the emitter, that is, there was not dispersion of the perturbation with the distance. That feature is theoretically explainable due to the coordinated dislocation of Cooper pairs (subjected to the same wave function) inside the boundary conditions imposed by the bulk of the superconducting disk (thickness and diameter) during the short time interval (time of high voltage discharge) in which the longitudinal force acted. Therefore, there is a perfect or almost perfect projection of the nonlocal induced force depending on the physical dimensions of the disk. Further according to the authors, there was an indication that the intensity of the impulse was proportional to the magnetic field magnitude in the interior of the superconductor and such a feature is consistent with the calculations here presented. Other relevant point is that simple calculations involving the deflexion of the pendulum indicate that the energy needed to a dislocation of $142 \mathrm{~mm}$ should be 2.14 times higher than the variation of potential energy indicated in Podkletnov work and that is consistent with our theoretical result, in which it is obtained a value 2.08 times higher. In relation to the indication of independence of the material used for the mass of the pendulum, the GQE hypothesis also is totally compatible and even reinforced.

\subsection{Experiments in Superconducting Toroids}

Our model can also explain the results reported in [2]. In that work, it was reported the presence of weak gravitation shielding properties of $\mathrm{YBa}_{2} \mathrm{Cu}_{3} \mathrm{O}_{7-\mathrm{y}}$ superconductor below $70 \mathrm{~K}$. We analyzed the results of that experiment and compared with our numerical results. Basically, the experimental apparatus consisted in a toroid with external radius $13.75 \mathrm{~cm}$ and internal one $4 \mathrm{~cm}$, total thickness $10 \mathrm{~mm}$, placed in the horizontal plane. The composite bulk was made by an upper layer of conducting ceramic with thickness 7 $\mathrm{mm}$ and a lower nonsuperconducting layer with $3 \mathrm{~mm}$. Three solenoids below the toroid were responsible by its levitation and two lateral solenoids were responsible by its rotation (all solenoids fed by alternate current). The test mass had values from $10 \mathrm{~g}$ up to $50 \mathrm{~g}$ and lost a percentage of weight when placed above the external border of the toroid (in the superconducting state below the critical temperature $70 \mathrm{~K}$ ) in levitation and rotation. In a rotation of $4000 \mathrm{rpm}$, a $50 \mathrm{~g}$ test mass lost $0.17 \%$ and the apparent weight loss increased proportionally to the rotation increasing. For $500 \mathrm{rpm}$, the same mass lost $0.23 \%$ of its weight. The article also indicates that the weight loss changes proportionally to the radial position of the test mass. In other words, the weight loss increases as function of the toroid radius.

We show in Figure 2 the scheme of the experimental apparatus implemented in that work.

The theoretical model here proposed indicates that it exists a nonlocal interaction (via generalized quantum entanglement) among Cooper pairs of the superconducting toroid in its superconducting phase and the particles composing the test mass placed above the toroid. As Cooper pairs are in the same quantum state they can be considered as a unique composed quantum particle and macroscopically spread in all the bulk of the toroid. The decreasing of the test mass weight loss is a kinetic consequence of Cooper pairs being subjected to the centrifugal force when the toroid is in rotation.

The calculation of the volume of the toroid is as follows:

$$
V_{1}=\pi r_{1}^{2} t
$$

in which $V_{1}$ is the volume of the solid toroid, $r_{1}$ is the large radius and $t$ is the thickness of the upper layer. So, we have:

$$
V_{1}=13.75^{2} \times 3.1417 \times 0.7=415.77 \mathrm{~cm}^{3}
$$

Analogously, we have

$$
V_{2}=\pi r_{2}^{2} t
$$

in which $V_{2}$ is the volume of the solid toroid, $r_{2}$ is the radius of the circular hole of the toroid and $t$ is the superconducting layer thickness. So:

$$
V_{2}=4^{2} \times 3.1417 \times 0.7=35.19 \mathrm{~cm}^{3}
$$




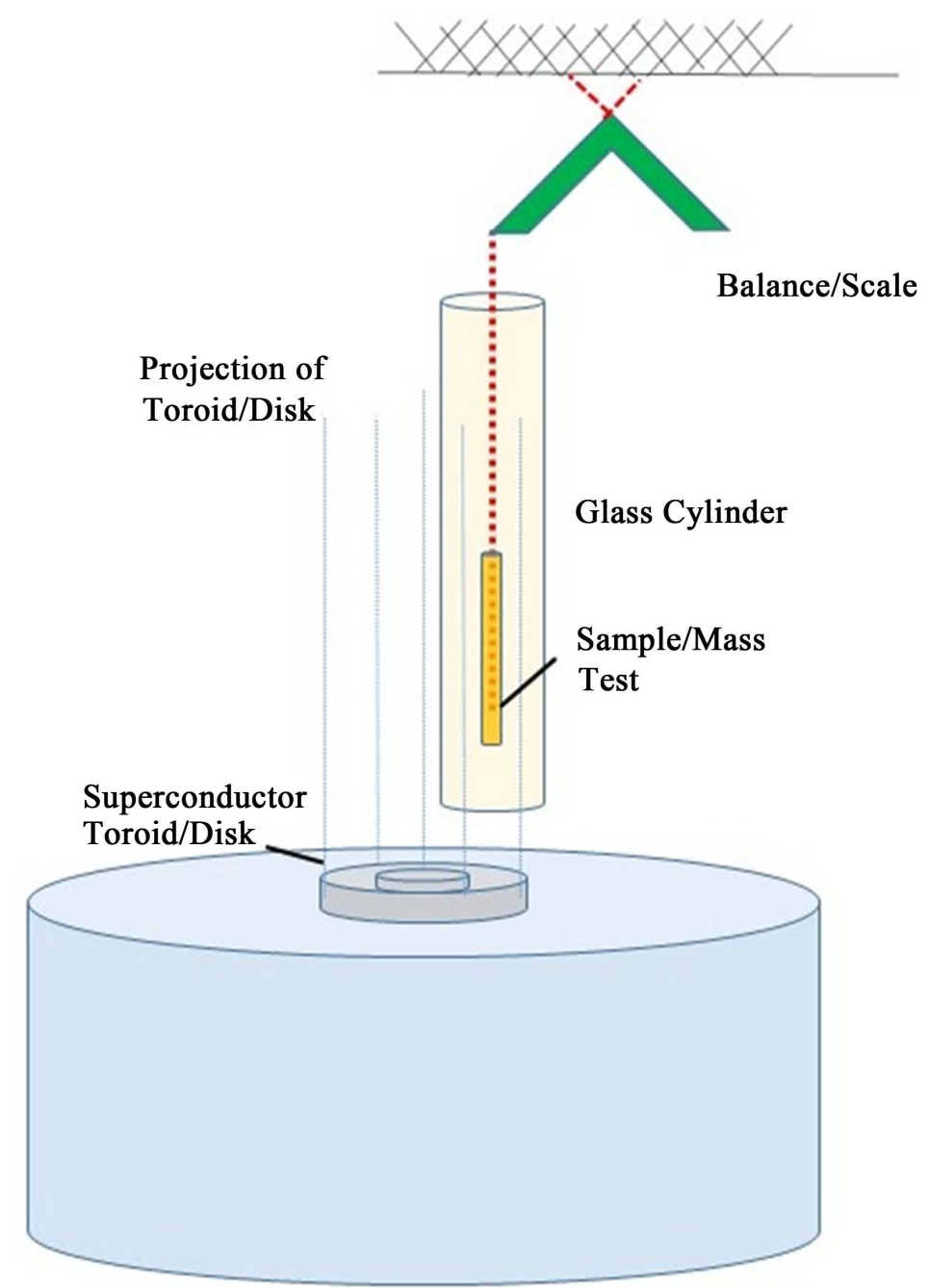

Figure 2. A simplified scheme of the experimental apparatus used in the experiments reported in [2]. The superconducting ceramic toroid was subjected to high currents and samples placed over it presented reduction of weight of up to $1.2 \%$, indicating the generation of a weak gravitational shielding in its surroundings.

As one has:

$$
V=V_{1}-V_{2}
$$

in which $V$ is the total volume of the toroid, then we have:

$$
V=415.77-35.19=380.58 \mathrm{~cm}^{3}
$$

The paper does not provide information about the density of electrons forming Cooper pairs in the superconducting ceramic in a determined temperature. The density of Cooper pairs in this material is lower than the values of the superconductors of type I. A typical value indicated in the literature is between $10^{21}$ and $5 \times 10^{21}$ electrons per cubic centimeter. In our choice, we consider the intermediate value one 2.5 $\times 10^{21}$ electrons per cubic centimeter. If we consider that value for the density of Cooper pairs and the electron mass $\left(9.1 \times 10^{-31} \mathrm{~kg}\right)$ we can calculate the total mass $m$ of Cooper pairs: 


$$
m=9.1 \times 10^{-31} \times 2.5 \times 10^{21} \times 380.58=8.66 \times 10^{-7} \mathrm{~kg}
$$

As Cooper pairs are subjected to a centrifuged force $F_{c}$ due to the rotation of the toroid, we calculated it in the model by considering

$$
F_{c}=m r w^{2}
$$

in which $m$ is the total mass of Cooper pairs, $r$ is the external radius of the toroid where the test mass is placed and $w$ is the angular velocity. Such a force is dependent on the radial position in relation to the axis of symmetry of the toroid in rotation. For an angular velocity of $4000 \mathrm{rpm}$, we have $w=4000 / 60=66.67 \mathrm{rad} / \mathrm{s}$, so that we obtain the force $F_{c}=8.66 \times 10^{-7} \times 13.75 \times 10^{-2} \times 66.67^{2}=5.29 \times 10^{-4} \mathrm{~N}$. The weight $P$ of the test mass $m_{P}=10 \mathrm{~g}$ is calculated by making $P=m_{P} \times g$, in which $m_{P}$ is the value of the test mass and $\mathrm{g}$ is the gravity acceleration. Inserting the values, one has $P=10 \times 10^{-3} \times 9.8=0.098 \mathrm{~N}$. The percentage weight loss of the test mass with $10 \mathrm{~g}$ can be calculated by:

$$
\Delta P=\frac{100 F_{c}}{P}
$$

So, we have $P=\left(5.29 \times 10^{-4} / 0.098\right) \times 100=0.534 \%$. For an angular velocity $5000 \mathrm{rpm}$, we have $W=$ $83.34 \mathrm{rad} / \mathrm{s}$. The centrifuged force for that angular velocity is

$$
F_{c}=8.66 \times 10^{-7} \times 13.75 \times 10^{-2} \times 83.34^{2}=8.25 \times 10^{-4} \mathrm{~N}
$$

The percentage weight loss of the test mass with $10 \mathrm{~g}$, in those conditions, is given by: $P=\left(8.25 \times 10^{-4} / 0.098\right) \times 100$ or $P=0.842 \%$. By considering an average test mass of $25 \mathrm{~g}$ (due to the limits of mass used in the experiment, e.g., $10 \mathrm{~g}$ and $50 \mathrm{~g}$ ), we respectively have the average percentage weight loss for $4000 \mathrm{rpm}$ and $5000 \mathrm{rpm}$ :

$$
P=\left(8.66 \times 10^{-7} \times 13.75 \times 10^{-2} \times 66.67^{2}\right) /\left(25 \times 10^{-3} \times 9.8\right) \times 100=0.216 \%
$$

and

$$
P=\left(8.66 \times 10^{-7} \times 13.75 \times 10^{-2} \times 83.34^{2}\right) /\left(25 \times 10^{-3} \times 9.8\right) \times 100=0.338 \%
$$

The values of weight loss of the test mass experimentally measured by considering the higher radius of the toroid $13.75 \mathrm{~cm}$ were found in the range from $0.3 \%$ and $0.5 \%$ for the angular velocities of $4000 \mathrm{rpm}$ and $5000 \mathrm{rpm}$, respectively. If we consider measurements made above the internal radius of the toroid (4.7 $\mathrm{cm})$, we have the following percentages of weight loss respectively calculated:

$$
P=\left(8.66 \times 10^{-7} \times 4.7 \times 10^{-2} \times 66.67^{2}\right) /\left(25 \times 10^{-3} \times 9.8\right) \times 100=0.07 \%
$$

and

$$
P=\left(8.66 \times 10^{-7} \times 4.7 \times 10^{-2} \times 83.34^{2}\right) /\left(25 \times 10^{-3} \times 9.8\right) \times 100=0.12 \%
$$

The values of weight loss experimentally measured by considering the higher value of the toroid radius $(13.75 \mathrm{~cm}$ ) were between $0.1 \%$ and $0.25 \%$ for the angular velocities $4000 \mathrm{rpm}$ and $5000 \mathrm{rpm}$, respectively. The values calculated in the model are close to the experimental values although the density of Cooper pairs is not so accurate. Besides another reason refers to the dependence on the value of the induced current, which is variable. The small differences in the decreasing of weight in the measurements of the different samples and the possible variations in the measurements as consequence of induced current variations can have suggested the error of considering that the values of test mass lost a same fraction of their weights. Losses of weight between $0.05 \%$ and $0.07 \%$ were measured by values of test mass above of the toroid in the superconducting toroid without angular velocity (static toroid only with levitation). The minimum magnetic force in order to have annulled the weight of Cooper pairs must be calculated by $P=$ $m \times g$, in which $m$ is the total mass of Cooper pairs and $g$ is the local gravity acceleration. So, we have: 


$$
P=9.1 \times 10^{-31} \times 5 \times 10^{21} \times 360.58 \times 9.8=1.6954 \times 10^{-5} \mathrm{~N}
$$

Such a repulsive force which Cooper pairs are subjected to be transmitted to the test mass of the toroid and is addressed upward (the direction of the magnetic levitation). The percentage weight loss for a test mass with $10 \mathrm{~g}$ placed above the toroid (any position over the area) is calculated as:

$$
P=\left(1.6954 \times 10^{-5} \mathrm{~N} \times 100\right) /\left(10 \times 10^{-3} \times 9.8\right)=0.0188 \%
$$

The best value of percentage measured in the experiments varied from $0.05 \%$ to $0.07 \%$, as indicated in the paper. The value calculated can converge to that range of values if we consider that the force of levitation is much higher than the weight of Cooper pairs because the toroid was hanged at height $3.5 \mathrm{~cm}$. For a magnitude of magnetic force necessary to suspend and make stable the toroid position at height 3.5 $\mathrm{cm}$-we estimate that such a force is three times higher than the weight of Cooper pairs-we obtained a percentage weight loss of the test mass with $10 \mathrm{~g}$ the value $P=0.0564 \%$, which is within the range measured in the experiments. It is remarkable that the centrifugal force is pointed radially from the center of symmetry axis to outer border of the toroid and this follows parallelly its horizontal surface but comparatively the force induced to the test mass is perpendicular. This can be explained considering the perpendicular deviation of Cooper pairs from the interface of lower conductor layer (resistive region) to the upper superconductor layer (no resistivity) [31], so that the nonlocal induced upward force pushes the test mass yielding its weight reduction.

As seen from the results, the effects are still weak for technological applications. However, in order to increase the magnitude of the effect so that it was strong enough to allow a practical application, we suppose that the anomalous effect could be enhanced if we consider some technological practices adopted in other physical situations, as suggested in [51], as to use time-dependent electric fields [52], introduce one (or many) impurity in the system [53], with pulsed magnetic fields [54-58], by making the superconductor rotate more quickly [59-61] or by using a superconductor device made of layers of different materials [62].

\section{CONCLUSIONS AND FINAL REMARKS}

In this work, we present a new model in order to theoretically explain in qualitative and quantitative terms, some experiments concerning to anomalous forces externally induced by superconductors built in different shapes and dimensions. The devices worked below the critical temperature in different conditions, that is, rotating in constant or variable angular velocity, levitating magnetically or over applying of high-voltage discharge. The different setups and experiments indicated weight losses in objects and unexplainable effects. For instance, either it was observed the reduction of weight in the case of superconducting toroids in rotation or the deflection of a simple pendulum with test mass composed by different dielectric materials, when it was close to superconducting disks subjected to high voltages.

It has been reported that a weak gravitational field was generated and detected in the neighborhood of superconducting ceramic disks that caused the deflection on the pendulum, but there was not still a definitive theory to explain such an anomalous effect. So, we here propose our model based on the same concept described in early similar works, which consists in attributing to generalized quantum entanglements the microscopic origin of the anomalous effect. As earlier considered, we can use classical analysis in the calculation of the physical quantities and show that the empirical formulas for the anomalous forces generated by the operation in high voltage of the superconductors provides very good values, in concordance with the experimental range measured for those experiments.

Our model considers a preexisting state of generalized quantum entanglement so that the Cooper pairs formed below the critical temperature of superconductors can exchange collectively their momentum variation with outer particles composing macroscopic objects such as the test mass of a simple pendulum or accelerometer. The good accordance of the magnitude of the induced nonlocal forces calculated from our model in comparison with the experimental values reinforces the concept of the application of classical quantities related to the macroscopic observables in the framework of GQE, such as described in other previous works in which other anomalous effects were analyzed. It is remarkable that the authors of the 
experiments mentioned in this work made a clear association of the phenomenon with the gravity for better explaining it. This argument reinforces the possible connection between the gravity and the preexisting condition of generalized quantum entanglement, that is, the gravity could be possibly understood in the context of generalized quantum nonlocal effects. Of course all those themes need more detailed analysis.

Finally, the present work really highlights the possibility that superconductor materials are applied to generate outer forces in the environment which could affect external neutral objects, that is, by another type of interaction different of the electromagnetic one. Rather it also reinforces our GQE theoretical framework in order to quantify the phenomena so that one encourages new experiments with superconductor ceramics. For example, new materials with higher density of Cooper pairs such as niobium can be applied in order to amplify the effects. In this way, although the phenomena demand special conditions, the effects can be enhanced enough to allow practical applications. Other possible ideas aiming to enhance the anomalous effects could be to adopt some technological practices, as for instance inserting a impurity in the materials, using time-dependent electric fields or designing new shapes or associating more devices or layers in the superconductors. As future perspective in such a topic, we aim to analyze the enhancement of the anomalous effect for materials with high density of current made of NbTi and other metallic superconductors. We are also analyzing how wide is the feature of dependence of the forces on the square of the current for other physical systems, as the semiconductor laser diodes, beside the case of superconductors here reported.

\section{REFERENCES}

1. Podkletnov, E. and Modanese, G. (2001) Impulse Gravity Generator Based on Charged $\mathrm{YBa}_{2} \mathrm{Cu}_{3} \mathrm{O}_{7-\mathrm{y}}$ Superconductor with Composite Crystal Structure. arXiv.Physics, Article ID: 0108005.

2. Podkletnov, E. (1997) Weak Gravitation Shielding Properties of Composite Bulk $\mathrm{YBa}_{2} \mathrm{Cu}_{3} \mathrm{O}_{7-\mathrm{y}}$ Superconductor below $70 \mathrm{~K}$ under e.m. Field. arXiv.Cond-Mat, Article ID: 9701074.

3. Podkletnov, E. and Modanese, G. (2003) Investigation of High Voltage Discharges in Low Pressure Gases through Large Ceramic Superconducting Electrodes. Journal of Low Temperature Physics, 132, 239-259. https://doi.org/10.1023/A:1024413718251

4. Lörincz, I. and Tajmar, M. (2015) Design and First Measurements of a Superconducting Gravity-Impulse-Generator. 51 st AIAA/SAE/ASEE Joint Propulsion Conference, AIAA Propulsion and Energy Forum, Orlando, 27-29 July 2015, 2015-4080.

5. Junker, T. (2012) Setup of the Impulse Gravity Generator Experiment Replication. In: Modanese, G., Robertson, G.A., Eds., Gravity-Superconductors Interaction: Theory and Experiment, Bentham Science Publishers, Sharjah, 183-202. https://doi.org/10.2174/978160805399511201010183

6. Solomon, B.T. (2011) Gravitational Acceleration without Mass and Noninertia Fields. Physics Essays, 24, 327-337. https://doi.org/10.4006/1.3595113

7. Solomon, B.T. (2012) An Introduction to Gravity Modification: A Guide to Using Laithwaite's and Podkletnov's Experiments and the Physics of Forces for Empirical Results. 2nd Edition, Universal Publishers, Irvine.

8. Solomon, B.T. (2013) New Evidence, Conditions, Instruments \& Experiments for Gravitational Theories. International Journal of Modern Physics, 4, 183-196. https://doi.org/10.4236/jmp.2013.48A018

9. Li, N., Noever, D., Robertson, T., Koczor, R. and Brantley, W. (1997) Static Test for a Gravitational Force Coupled to Type II YBCO Superconductors. Physica C, 281, 260-267.

https://doi.org/10.1016/S0921-4534(97)01462-7

10. Bull, M. and De Podesta, M. (1995) Alternative Explanation of "Gravitational Screening” Experiments. Physica C, 253, 199-200. https://doi.org/10.1016/0921-4534(95)00494-7

11. Unnikrishnan, C.S. (1996) Does a Superconductor Shield Gravity? Physica C, 266, 133. 
https://doi.org/10.1016/0921-4534(96)00340-1

12. DeWitt, B.S. (1966) Superconductors and Gravitational Drag. Physical Review Letters, 16, 1092-1093. https://doi.org/10.1103/PhysRevLett.16.1092

13. Papini, G. (1967) Detection of Inertial Effects with Superconducting Interferometers. Physics Letters A, 24, 32-33. https://doi.org/10.1016/0375-9601(67)90178-8

14. Felch, S.B., Tate, J., Cabrera, B. and Anderson, J.T. (1985) Precise Determination of $h / m_{e}$ Using a Rotating, Superconducting Ring. Physical Review B, 31, 7006-7011. https://doi.org/10.1103/PhysRevB.31.7006

15. Anandan, J. (1994) Relativistic Gravitation and Superconductors. Classical and Quantum Gravity, 11, A23-A38. https://doi.org/10.1088/0264-9381/11/6A/003

16. Anandan, J. (1979) Interference, Gravity and Gauge Fields. II Nuovo Cimento A, 53, 221-250. https://doi.org/10.1007/BF02776416

17. Anandan, J. (1984) Relativistic Thermoelectromagnetic Gravitational Effects in Normal Conductors and Superconductors. Physics Letters A, 105, 280-284. https://doi.org/10.1016/0375-9601(84)90997-6

18. Ross, D.K. (1983) The London Equations for Superconductors in a Gravitational Field. Journal of Physics A: Mathematical and General, 16, 1331-1336. https://doi.org/10.1088/0305-4470/16/6/026

19. Hirakawa, H. (1975) Superconductors in Gravitational Field. Physics Letters A, 53, 395-396. https://doi.org/10.1016/0375-9601(75)90045-6

20. Rystephanick, R.G. (1973) On the London Moment in Rotating Superconducting Cylinders. Canadian Journal of Physics, 51, 789-794. https://doi.org/10.1139/p73-107

21. Peng, H., Lind, G. and Chin, Y.S. (1991) Interaction between Gravity and Moving Superconductors. General Relativity and Gravitation, 23, 1231-1250. https://doi.org/10.1007/BF00756846

22. Ciubotariu, C. and Agop, M. (1996) Absence of a Gravitational Analog to the Meissner Effect. General Relativity and Gravitation, 28, 405-412. https://doi.org/10.1007/BF02105084

23. Agop, M., Buzea, C.G., Griga, V., Ciubotariu, C., Buzea, C., Sta, C. and Jatomir, D. (1996) Gravitational Paramagnetism, Diamagnetism and Gravitational Superconductivity. Australian Journal of Physics, 49, 1063-1074. https://doi.org/10.1071/PH961063

24. Li, N. and Torr, D.G. (1991) Effects of a Gravitomagnetic Field on Pure Superconductors. Physical Review D, 43, 457-459. https://doi.org/10.1103/PhysRevD.43.457

25. Peng, H., Torr, D.G., Hu, E.K. and Peng, B. (1991) Electrodynamics of Moving Superconductors and Superconductors under the Influence of External Forces. Physical Review B, 43, 2700-2704. https://doi.org/10.1103/PhysRevB.43.2700

26. Li, N. and Torr, D.G. (1992) Gravitational Effects on the Magnetic Attenuation of Superconductors. Physical Review B, 46, 5489-5495. https://doi.org/10.1103/PhysRevB.46.5489

27. Torr, D.G. and Li, N. (1993) Gravitoelectric-Electric Coupling via Superconductivity. Foundations of Physics Letters, 6, 371-383. https://doi.org/10.1007/BF00665654

28. Modanese, G. (1996) Theoretical Analysis of a Reported Weak-Gravitational-Shielding Effect. Europhysics Letters, 35, 413-418. https://doi.org/10.1209/epl/11996-00129-8

29. Agop, M., Buzea, C.G. and Nica, P. (2000) Local Gravitoelectromagnetic Effects on a Superconductor. Physica C, 15, 120. https://doi.org/10.1016/S0921-4534(00)00340-3

30. Agop, M., Ioannou, P.D. and Diaconu, F. (2000) Some Implications of Gravitational Superconductivity. Progress of Theoretical Physics, 104, 733-742. https://doi.org/10.1143/PTP.104.733

31. Misner, C.W., Thorne, K.S. and Wheeler, J.A. (1973) Gravitation. W. H. Freeman and Company. 
32. Braginsky, V.B., Caves, C.M. and Thorne, K.S. (1977) Laboratory Experiments to Test Relativistic Gravity. Physical Review D, 15, 2047-2068. https://doi.org/10.1103/PhysRevD.15.2047

33. Modanese, G. (2013) A Comparison between the YBCO Discharge Experiments by E. Podkletnov and C. Poher, and Their Theoretical Interpretations. Applied Physics Research, 5, 59-73. https://doi.org/10.5539/apr.v5n6p59

34. Modanese, G. and Fontana, G. (2004) Effect of the Vacuum Energy Density on Graviton Propagation, CP699, Space Technology and Applications International, Forum-STAIF. AIP Proceedings.

35. Modanese, G. (2007) The Vacuum State of Quantum Gravity Contains Large Virtual Masses. Classical and Quantum Gravity, 24, 1899-1910. https://doi.org/10.1088/0264-9381/24/8/001

36. Modanese, G. and Junker, T. (2008) Conditions for Stimulated Emission in Anomalous Gravity-Superconductors Interactions. In: Christiansen, M.N. and Rasmussen, T.K., Eds., Classical and Quantum Gravity Research, Nova Science Publishers, 245-269.

37. Modanese, G. (2012) Anomalous Gravitational Vacuum Fluctuations Which Act as Virtual Oscillating Dipoles. arXiv:1110.2059.

38. Atanasov, V. (2017) The Geometric Field (Gravity) as an Electro-Chemical Potential in a Ginzburg-Landau Theory of Superconductivity. Physica B, 517, 3-58. https://doi.org/10.1016/j.physb.2017.05.006

39. Ummarino, G.A. and Gallerati, A. (2017) Superconductor in a Weak Static Gravitational Field. The European Physical Journal C, 77, 1-12. https://doi.org/10.1140/epjc/s10052-017-5116-y

40. Porcelli, E.B. and Filho, V.S. (2016) On the Anomalous Forces in High-Voltage Symmetrical Capacitors. Physics Essays, 29, 2-9. https://doi.org/10.4006/0836-1398-29.1.002

41. Porcelli, E.B. and Filho, V.S. (2015) On the Anomalous Weight Losses in High-Voltage Symmetrical Capacitors. arXiv:1502.06915.

42. Porcelli, E.B. and Filho, V.S. (2016) Characterisation of Anomalous Asymmetric High-Voltage Capacitors. IET Science, Measurement \& Technology, 10, 383-388. https://doi.org/10.1049/iet-smt.2015.0250

43. Porcelli, E.B. and Filho, V.S. (2017) Anomalous Effects from Dipole-Environment Quantum Entanglement. International Journal of Advance Engineering Research and Science, 4, 131-144.

https://doi.org/10.22161/ijaers.4.1.21

44. Porcelli, E.B. and Filho, V.S. (2016) Induction of Force Performed by Piezoelectric Materials. arXiv:1612.04201.

45. Porcelli, E.B. and Filho, V.S. (2017) Induction of Forces at Distance Performed by Semiconductor Laser Diodes. American Journal of Engineering Research, 6, 35-48.

46. Bueno, M. and Assis, A.K.T. (2015) Cálculo de Indutância e de Força em Circuitos Elétricos. [Calculation of Inductance and Force between Electric Circuits.] 2nd Edition, Roy Keys Inc.

47. Dagotto, E. (1994) Correlated Electrons in High-Temperature Superconductors. Reviews of Modern Physics, 66, 763-840. https://doi.org/10.1103/RevModPhys.66.763

48. Van Harlingen, D.J. (1995) Phase-Sensitive Tests of the Symmetry of the Pairing State in the High-Temperature Superconductors-Evidences for Symmetry $\mathrm{d}_{\mathrm{x}-\mathrm{y}}^{2}{ }^{2}$. Reviews of Modern Physics, 67, 515-535. https://doi.org/10.1103/RevModPhys.67.515

49. Cho, A. (2012) A Final Answer on How High-Temperature Superconductors Don't Work? http://www.sciencemag.org/author/adrian-cho

50. Tavkhelidze, A.A. (2011) Mechanism of Cooper-Pairing in Layered High Temperature Superconductors. arxiv:1109.3978.

51. Ummarino, G.A. (2001) Possible Alterations of the Gravitational Field in a Superconductor. arXiv:cond-mat/0010399v3. 
52. Konsin, P. and Sorkin, B. (1998) Electric Field Effects in High- $\mathrm{T}_{\mathrm{c}}$ Cuprates. Physical Review B, 58, 5795-5802. https://doi.org/10.1103/PhysRevB.58.5795

53. Krempasky, J.J. and Thomson, R.S. (1985) Time-Dependent Ginzburg-Landau Equations for a Dirty Gapless Superconductor. Physical Review B, 32, 2965-2975. https://doi.org/10.1103/PhysRevB.32.2965

54. Kaper, H.G. and Takac, P. (1998) Ginzburg-Landau Dynamics with a Time-Dependent Magnetic Field. Nonlinearity, 11, 291-306. https://doi.org/10.1088/0951-7715/11/2/006

55. Dorsey, A.T. (1995) Linear Response of Thin Superconductors in Perpendicular Magnetic Fields: An Asymptotic Analysis. Physical Review B, 51, 15329-15343. https://doi.org/10.1103/PhysRevB.51.15329

56. Gurevich, A. and Brandt, E.H. (1997) AC Response of Thin Superconductors in the Flux-Creep Regime. Physical Review B, 55, 12706-12718. https://doi.org/10.1103/PhysRevB.55.12706

57. Kozhevnikov, A.A. (1995) Production of a Magnetic Flux Vortex in a Time-Dependent Field. Physics Letters A, 202, 343-346. https://doi.org/10.1016/0375-9601(95)00353-5

58. Vlasko-Vlasov, V.K., Welp, U., Crabtree, G.W., Gunter, D., Kabanov, V.V., Nikitenko, V.I. and Paulius, L.M. (1998) Meissner Holes and Turbulent Structures in Superconductors in Unidirectional and Rotating Fields. Physical Review B, 58, 3446-3456. https://doi.org/10.1103/PhysRevB.58.3446

59. Liu, M. (1998) Rotating Superconductors and the Frame-Independent London Equation. Physical Review Letters, 81, 3223-3226. https://doi.org/10.1103/PhysRevLett.81.3223

60. Carter, B., Prix, R. and Langlois, D. (2000) Energy of Magnetic Vortices in a Rotating Superconductor. Physical Review B, 62, 9740-9747. https://doi.org/10.1103/PhysRevB.62.9740

61. Geurst, J.A. (1980) Unifying Phenomenological Approach to the London and Ginzburg-Landau Theories of Superconductivity in the General Unsteady Case. Physica B, 101, 82-97. https://doi.org/10.1016/0378-4363(80)90090-X

62. Lipowsky, R. and Speth, W. (1983) Semi-Infinite Systems with First-Order Bulk Transitions. Physical Review B, 28, 3983-3993. https://doi.org/10.1103/PhysRevB.28.3983

\section{Scientific Research Publishing}

\section{Submit or recommend next manuscript to SCIRP and we will provide best service for you:}

Accepting pre-submission inquiries through Email, Facebook, LinkedIn, Twitter, etc.

A wide selection of journals (inclusive of 9 subjects, more than 200 journals)

Providing 24-hour high-quality service

User-friendly online submission system

Fair and swift peer-review system

Efficient typesetting and proofreading procedure

Display of the result of downloads and visits, as well as the number of cited articles

Maximum dissemination of your research work

Submit your manuscript at: http://papersubmission.scirp.org/

Or contact ns@scirp.org 